\title{
The Obesity Paradox for Stroke Patients, a Back Side of the
}

\section{Coin}

\section{Sonmezler A*}

Department of Neurology, Adana City Research and Training Hospital, Turkey

*Corresponding author: Abdurrahman Sonmezler, Department of Neurology, Adana

City Research and Training Hospital, Adana-Turkey, Email: sonmezlera@gmail.com

\section{Mini Review \\ Volume 4 Issue 1}

Received Date: April 09, 2019

Published Date: May 08, 2019

\section{Abstract}

A high BMI (body mass index) has been associated with higher rates of survival in patients with cardiovascular disease including patients with cerebrovascular disease, which is referred to as the obesity paradox. But the back of the coin explains the subject. In fact, body mass index does not fully reflect visceral adiposity, which is the cause of risk. In conclusion, it should be used with other parameters that show visceral adiposity to reveal the risk of obesity, metabolic syndrome and cerebrovascular disease.

Keywords: Body Mass Index; Visceral adiposity; Stroke patient

Abbreviations: BMI: Body Mass İndex; VAD: Visceral Adipose Dysfunction; VAT: Visceral Adipose Tissue; HDL: High Density Lipoprotein.

\section{Mini Review}

The fat accumulated in the body is collected either under the skin or in the abdomen. Visceral fat accumulation, known as central or abdominal fat accumulation, is the most dangerous type of fat accumulation. Because it plays a role in the formation of cardiovascular diseases, diabetes and other chronic diseases. This table, known as metabolic syndrome, involves serious health risks. Visceral fat typically comprises a small percentage of total body fat, but has serious effects on health. This fat accumulates in the body is considered an endocrine organ. Because it is metabolically active, it is affected by hormones. It also secretes a large number of hormone-like proteins and strong chemicals. The fat collected in the abdomen is particularly dangerous because it is metabolically very active, leads to inflammation in the body, and initiates processes leading to diseases at the cell level [1-4].
Metabolic syndrome (MetS) is known to be a cluster of interrelated risk factors of metabolic origin such as elevated blood pressures, glucose metabolism disturbances, dyslipidemia, and obesity which are linked to the development of atherosclerotic cardiovascular diseases and type 2 diabetes mellitus. Of these risk factors, obesity or body fat seems to be the predominant underlying risk factor not only in the development of MetS but also other cardiovascular risk factors. Obesity is important because it causes metabolic syndrome and atherosclerosis.Waist circumference, body mass index, waist-to-height ratio a body shape index, body roundness index, visceral adiposity index, abdominal volume index, Conicity Index , body adiposity index, lipid accumulation product and waist circumference triglyceride index anthropometric parameters are predict Metabolic Syndrome [5].

Obesity and blood lipids are closely related to cardiovascular disease and diabetes. Various obesity and lipid-related parameters, especially body mass index, waist-to-height ratio, triglyceride-to-high-densitylipoprotein cholesterol, lipid accumulation product and visceral Adiposity index, have commonly been used to predict MetS. BMI is a measurement of body fat based on 


\section{Anaesthesia \& Critical Care Medicine Journal}

height and weight., waist-to-height ratio reflects abdominal or central obesity [6].

The Visceral Adipozity Index (VAI) is a mathematical model that uses both anthropometric (body mass index [BMI] and WC) and functional (triglycerides [TG] and high density lipoprotein [HDL] cholesterol) simple parameters. This index, which can be considered as a simple marker of visceral adipose dysfunction (VAD), showed a strong relationship with both the use of peripheral glucose (M value) and visceral adipose tissue (VAT) as measured by MRI. It showed a strong and independent relationship with both cardiovascular and cerebrovascular events [7]. Mortality is lower and clinical outcomes are better for stroke patients with a high body mass index (BMI) who are classified as obese or overweight, which has been termed the obesity paradox. The most important limitations associated with BMI are its inability to distinguish muscle tissue from body fat, as well as to reflect mass distribution and body composition. Accounting for body fat distribution is important because visceral fat accumulation is strongly associated with a higher cerebrometabolic risk. Although subcutaneous adiposity confers a reduced cerebrovascular and metabolic risk compared with the same proportion of visceral abdominal fat, all these patients are classified into the same obesity category on the basis of their BMI [8].

Obesity is evaluated by various methods such as body mass index (BMI), waist-to-hip ratio, and visceral-tosubcutaneous fat ratio (VS ratio). A previous study showed the clinical significance of BMI as a predictor of mortality or morbidity. Furthermore, waist-to-hip ratio, a trait of $9 * 9$ the criteria for metabolic syndrome, is more strongly related to cardiovascular disease than BMI. Measurement of visceral fat area is also reported to be useful for predicting atherosclerosis because adipose tissues in the visceral fat endocrine substances progress atherosclerosis. Higuchi and colagues' study found that VS ratio was independently related to small and large cerebrovascular lesions. Although the their study included apparently healthy subjects with few classic risk factors of atherosclerosis, cerebral ischemic changes and carotid intimal thickness were observed more than expected. Visceral fat accumulation may progress cerebrovascular lesions before subjects develop multiple risk factors of atherosclerosis. Visceral fat was independently associated with both small and large vessel lesions in apparently healthy subjects, but BMI and waistto-hip ratio were not. Visceral fat and its ratio to subcutaneous fat might be a keen predictor of cerebrovascular lesions even in healthy subjects [9].
The obesity paradox remain debatable, partially due to methodological issues and the inherent limitations of BMI as a measure of adiposity. BMI cannot distinguish between sources (i.e. muscle or adipose tissue) or locations of body mass (i.e. gluteal-femoral or abdominal regions) [10]. As can be seen, studies based on BMI alone can produce controversial results. The obesity paradox is also demonstrated in this way.Consequently, it should be used with other parameters that show visceral adiposity, not body mass index alone, to reveal the risk of obesity, metabolic syndrome,cerebrovascular disease.

\section{References}

1. Andersen KK, Olsen TS (2015) The obesity paradox in stroke: lower mortality and lower risk of readmission for recurrent stroke in obese stroke patients. Int J Stroke 10(1): 99-104.

2. Bastard JP, Maachi M, Lagathu C, Kim MJ, Caron M, et al. (2006) Recent advances in the relationship between obesity, inflammation and insulin resistance. Eur Cytokine Netw 17(1): 4-12.

3. Galletti F, Barbato A, Versiero M, Iacone R, Russo O (2007) Circulating leptin levels predict the development of metabolic syndrome in middle-aged men: an 8 year follow-up study. J Hypert 25(8): 16711677.

4. De Lorenzo A, Del Gobbo V, Premrov MG, Bigioni M, Galvano F, et al. (2007) Normal-weight obese syndrome: early inflammation? Am J Clin Nutr 85(1): 40-45.

5. Adejumo EN, Adejumo AO, Azenabor A, Ekun AO, Enitan SS, et al. (2019) Anthropometric parameter that best predict metabolic syndrome in South west Nigeria. Diabetes \& Metabolic Syndrome: Clinical Research \& Reviews 13(1): 48-54.

6. Gu Z, Zhu P, Wang Q, He H, Xu J, et al. (2018) Obesity and lipid-related parameters for predicting metabolic syndrome in Chinese elderly population. Lipids Health Dis 17(1): 289.

7. Marco C Amato, Carla Giordano, Maria Pitrone, Aldo Galluzzo (2011) Cut-off points of the visceral adiposity index (VAI) identifying a visceral adipose dysfunction associated with cardiometabolic risk in a Caucasian Sicilian population. Lipids in Health and Disease 10: 183. 


\section{Anaesthesia \& Critical Care Medicine Journal}

8. Ja-Hae Kim, Kang-Ho Choi, Kyung-Wook Kang, JoonTae Kim, Seong-Min Choi, et al. (2019) Impact of Visceral Adipose Tissue on Clinical Outcomes After Acute Ischemic Stroke. Stroke 50: 448-454.

9. Satoshi Higuchi, Yusuke Kabeya, Kiyoe Kato (2017) Visceral-to-subcutaneous fat ratio is independently related to small and large cerebrovascular lesions even in healthy subjects. Atherosclerosis 259: 41-45.
10. Jaspers NEM, Dorresteijn JAN, van der Graaf Y, Westerink J, Kappelle LJ, et al. (2017) Visseren, on behalf of the SMART Study Group, Relation between adiposity and vascular events,malignancy, and mortality in patients with stable cerebrovascular disease. International Journal of Obesity 41(12): 1775-1781. 